\title{
DERIVATION OF DIRAC'S EQUATION FROM THE EVANS WAVE EQUATION
}

\author{
M. W. Evans \\ Alpha Institute for Advanced Study \\ E-mail: emyrone@aol.com
}

Received 27 August 2003; revised 25 October 2003

The Evans wave equation [1] of general relativity is expressed in spinor form, thus producing the Dirac equation in general relativity. The Dirac equation in special relativity is recovered in the limit of Euclidean or flat spacetime. By deriving the Dirac equation from the Evans equation it is demonstrated that the former originates in a novel metric compatibility condition, a geometrical constraint on the metric vector $q_{\mu}$ used to define the Einstein metric tensor. Contrary to some claims by Ryder, it is shown that the Dirac equation cannot be deduced unequivocally from a Lorentz boost in special relativity. It is shown that the usually accepted method in Clifford algebra and special relativity of equating the outer product of two Pauli spinors to a three-vector in the Pauli basis leads to the paradoxical result $X=Y=Z=0$. The method devised in this paper for deriving the Dirac equation from the Evans equation does not use this paradoxical result.

Key words: Evans wave equation, general relativity, Dirac equation in general and special relativity, metric four-vector.

\section{INTRODUCTION}

A novel and fundamental wave equation of general relativity can be deduced [1] from the metric compatibility condition

$$
D_{\mu} q^{\nu}=\partial_{\mu} q^{\nu}+\Gamma_{\mu \lambda}^{\nu} q^{\lambda}=0
$$

on the metric four-vector $q_{\mu}$ used in the standard definition [2,3] of the metric tensor of the Einstein field equation

$$
q_{\mu \nu}=q_{\mu} q_{\nu}
$$


as the outer product of two metric vectors. Equation (1) states that the covariant derivative of the metric vector $q^{\nu}$ vanishes. It has been shown that the metric compatibility condition (1) leads to the usual metric compatibility condition of the metric tensor

$$
D_{\sigma} q_{\mu \nu}=0
$$

used in Riemann geometry [3] to relate the Christoffel symbol $\Gamma_{\mu \lambda}^{\nu}$ and metric tensor $q_{\mu \nu}$. Covariant differentiation [4] of the compatibility condition (1) leads to the Evans wave equation $[1,4]$

$$
(\square+k T) q^{\mu}=0 .
$$

In Eq. (4), $T$ is the standard contracted form of the canonical energy momentum tensor

$$
T=q^{\mu \nu} T_{\mu \nu}
$$

$k$ is the Einstein constant, and $\square$ the flat spacetime d'Alembertian operator.

In Sec. 2 the Dirac equation in general relativity is obtained from Eq. (4) by expressing the metric three vector in non-Euclidean spacetime as a two-component metric spinor, then applying the parity operation to the metric spinor to obtain a metric spinor with four components. This procedure produces the Dirac equation in general relativity. In Sec. 3 the well known Dirac equation of special relativity is obtained as a limiting form of Eq. (4) defined by

$$
k T \rightarrow m^{2} c^{2} / \hbar^{2},
$$

where $m$ denotes mass, $c$ the speed of light in vacuo, and $\hbar$ the Dirac constant. In Sec. 4 it is shown that the Dirac equation in general relativity can also be written as

$$
(\square+k T) \gamma^{\mu}=0,
$$

where $\gamma^{\mu}$ is the Dirac matrix generalized to non-Euclidean spacetime, a $4 \times 4$ matrix related to the tetrad. The most general form of Eq. (4) is $[1,2]$

$$
(\square+k T) q_{\mu}^{a}=0,
$$

where $q_{\mu}^{a}$ is the tetrad [3].

In Sec. 5 it is shown that the accepted method [5] in Clifford algebra in special (and also general) relativity of relating the components of a three-vector to those of a two-spinor leads to a fundamental paradox of Clifford algebra

$$
X=Y=Z=0 .
$$


Therefore we do not use this paradoxical but unfortunately well accepted method in our derivations in this paper. We devise a simple and correct method Eq. (28) for relating the three vector components to the two Pauli spinor components.

Finally it is shown that the Dirac equation cannot be derived unequivocally from a Lorentz boost in special relativity. The correct way to derive the Dirac equation is from non-Euclidean geometry in general relativity, using the metric four vector $q_{\mu}$. We refer to Eq. (4) as the "Evans equation" for ease of reference.

\section{DERIVATION OF THE DIRAC EQUATION OF GENERAL RELATIVITY}

We start the derivation by considering the ordinary position vector $\mathbf{R}$ in three dimensional space and correctly deriving the Pauli spinor from $\mathbf{R}$. The method thus developed is used then to construct the metric spinor from the metric three-vector in non-Euclidean spacetime

of general relativity. This allows the metric three-vector $\mathbf{q}$ in nonEuclidean spacetime to be expressed as a metric two-spinor

$$
q=\left(\begin{array}{l}
q_{1} \\
q_{2}
\end{array}\right) .
$$

Finally the parity operation is applied to the two-spinor to produce a metric four-spinor $\psi$ :

$$
\psi=\left(\begin{array}{c}
q_{1}^{(R)} \\
q_{2}^{(R)} \\
q_{1}^{(L)} \\
q_{2}^{(L)}
\end{array}\right)=\left(\begin{array}{l}
q^{(R)} \\
q^{(L)}
\end{array}\right)
$$

where the superscripts $(R)$ and $(L)$ denote right-handed and lefthanded two-spinors, respectively. The Dirac equation in general relativity is then deduced to be

$$
(\square+k T) \psi=0 .
$$

The position vector $\mathbf{R}$ is defined in the Cartesian basis in standard notation $[2]$

$$
\mathbf{R}=X \hat{\mathbf{i}}+Y \hat{\mathbf{j}}+Z \hat{\mathbf{k}}
$$


In the Pauli basis [5], the position vector is

$$
r=\boldsymbol{\sigma} \cdot \mathbf{r}=X \sigma_{1}+Y \sigma_{2}+Z \sigma_{3}=\left(\begin{array}{cc}
Z & X-i Y \\
X+i Y & -Z
\end{array}\right)
$$

and the square of the position vector is the invariant

$$
\begin{aligned}
r^{2}=(\boldsymbol{\sigma} \cdot \mathbf{r})(\boldsymbol{\sigma} \cdot \mathbf{r}) & =\left(\begin{array}{cc}
Z & X-i Y \\
X+i Y & -Z
\end{array}\right)\left(\begin{array}{cc}
Z & X-i Y \\
X+i Y & -Z
\end{array}\right) \\
& =\left(X^{2}+Y^{2}+Z^{2}\right)\left(\begin{array}{ll}
1 & 0 \\
0 & 1
\end{array}\right) .
\end{aligned}
$$

The four Pauli matrices

$$
\begin{array}{ll}
\sigma_{0}=\left(\begin{array}{ll}
1 & 0 \\
0 & 1
\end{array}\right), \quad \sigma_{1}=\left(\begin{array}{ll}
0 & 1 \\
1 & 0
\end{array}\right), \\
\sigma_{2}=\left(\begin{array}{cc}
0 & -i \\
i & 0
\end{array}\right), \quad \sigma_{3}=\left(\begin{array}{cc}
1 & 0 \\
0 & -1
\end{array}\right),
\end{array}
$$

are all Hermitian and unitary. However, while the determinant of the unit matrix $\sigma_{0}$ is +1 , the determinants of the other three Pauli matrices are -1 in each case. The latter therefore do not belong to the group $S U(2)$. But this does not affect their $S U(2)$ commutation symmetry. The $S U(2)$ group is commonly defined as consisting of all unitary, unimodular $2 \times 2$ matrices:

$$
U U^{\dagger}=1, \quad \operatorname{det} U=1 .
$$

They all have the general form

$$
U=\left(\begin{array}{cc}
a & b \\
-b^{*} & a^{*}
\end{array}\right)
$$

subject to the constraint

$$
a a^{*}+b b^{*}=1 .
$$

Under an $S U(2)$ transformation, the following occurs:

$$
\xi^{\prime}=U \xi, \quad \text { i.e., } \quad\left(\begin{array}{l}
\xi_{1}^{\prime} \\
\xi_{2}^{\prime}
\end{array}\right)=\left(\begin{array}{cc}
a & b \\
-b^{*} & a^{*}
\end{array}\right)\left(\begin{array}{l}
\xi_{1} \\
\xi_{2}
\end{array}\right),
$$




$$
\xi^{+\prime}=\xi^{+} U^{+}, \quad \text { i.e., } \quad\left(\xi_{1}^{* \prime}, \xi_{2}^{* \prime}\right)=\left(\xi_{1}^{*}, \xi_{2}^{*}\right)\left(\begin{array}{c}
a^{*}-b \\
b^{*} a
\end{array}\right)
$$

where

$$
\xi=\left(\begin{array}{c}
\xi_{1} \\
\xi_{2}
\end{array}\right), \quad \xi^{+}=\left(\xi_{1}^{*}, \xi_{2}^{*}\right)
$$

is the two-component spinor with complex-valued elements. The twospinor is often referred to as the Pauli spinor but was devised in the nineteenth century by Clifford. Therefore, under a $S U(2)$ transformation, we obtain four equations

$$
\begin{aligned}
\xi_{1}^{\prime} & =a \xi_{1}+b \xi_{2}, \\
\xi_{2}^{\prime} & =-b^{*} \xi_{1}+a^{*} \xi_{2}, \\
\xi_{1}^{* \prime} & =\xi_{1}^{*} a^{*}+\xi_{2}^{*} b^{*}, \\
\xi_{2}^{* \prime} & =-b \xi_{1}^{*}+a \xi_{2}^{*} .
\end{aligned}
$$

Consider the inner products

$$
\begin{aligned}
& R^{2}=(X, Y, Z)\left(\begin{array}{c}
X \\
Y \\
Z
\end{array}\right)=X^{2}+Y^{2}+Z^{2}, \\
& R^{2}=\left(\xi_{1}^{*}, \xi_{2}^{*}\right)\left(\begin{array}{l}
\xi_{1} \\
\xi_{2}
\end{array}\right)=\xi_{1} \xi_{1}^{*}+\xi_{2} \xi_{2}^{*}
\end{aligned}
$$

to obtain the simple invariant

$$
R^{2}=X^{2}+Y^{2}+Z^{2}=\xi_{1} \xi_{1}^{*}+\xi_{2} \xi_{2}^{*} .
$$

This is a relation between the three real-valued components $X, Y$ and $Z$ of the three-vector, and the two complex-valued components $\xi_{1}$ and $\xi_{2}$ of the two-spinor. Define $O(3)$ as the group of rotations in threedimensional space [5]. Under an $O(3)$ transformation,

$$
R^{2}=X^{2}+Y^{2}+Z^{2}=X^{\prime 2}+Y^{\prime 2}+Z^{\prime 2} .
$$

Similarly, under an $S U(2)$ transformation,

$$
R^{2}=\xi_{1} \xi_{1}^{*}+\xi_{2} \xi_{2}^{*}=\xi_{1}^{\prime} \xi_{1}^{* \prime}+\xi_{2}^{\prime} \xi_{2}^{* \prime} .
$$

If we choose $b=0$ in the constraint condition (19), then

$$
a a^{*}=1 ;
$$


and, from Eqs. (31), (30), (24), and (23):

$$
R^{2}=a a^{*} \xi_{1} \xi_{1}^{*}+a^{*} a \xi_{2} \xi_{2}^{*}=\xi_{1} \xi_{1}^{*}+\xi_{2} \xi_{2}^{*},
$$

as required. Therefore

$$
a a^{*}=1, \quad b b^{*}=0
$$

and

$$
U=\left(\begin{array}{cc}
a & 0 \\
0 & a^{*}
\end{array}\right)
$$

is one possible representation of $U$, the $S U(2)$ transformation matrix.

Therefore, Eqs. (20) and (21) become

$$
\left(\begin{array}{l}
\xi_{1}^{\prime} \\
\xi_{2}^{\prime}
\end{array}\right)=\left(\begin{array}{cc}
a & 0 \\
0 & a^{*}
\end{array}\right)\left(\begin{array}{l}
\xi_{1} \\
\xi_{2}
\end{array}\right), \quad\left(\xi_{1}^{* \prime}, \xi_{2}^{* \prime}\right)=\left(\xi_{1}^{*}, \xi_{2}^{*}\right)\left(\begin{array}{cc}
a^{*} & 0 \\
0 & a
\end{array}\right) .
$$

If $b=b^{*}=0$, Eq. (35) gives Eqs. (23) and (24), as required.

In order to build up the Dirac equation in general relativity from the Evans equation the above derivations based on the position vector $\mathbf{R}$ in flat (or Euclidean) spacetime must be repeated for the metric vector q of non-Euclidean spacetime in its spinor representation in non-Euclidean spacetime.

First, consider the metric three-vector in Euclidean spacetime:

$$
\mathbf{q}=q_{X} \mathbf{i}+q_{Y} \mathbf{j}+q_{Z} \mathbf{k} .
$$

Then

$$
q^{2}=q_{X}^{2}+q_{Y}^{2}+q_{Z}^{2}
$$

is invariant under an $O(3)$ transformation. Define the metric spinor

$$
q=\left(\begin{array}{c}
q_{1} \\
q_{2}
\end{array}\right), \quad q^{+}=\left(q_{1}^{*}, q_{2}^{*}\right),
$$

such that

$$
q^{2}=q_{X}^{2}+q_{Y}^{2}+q_{Z}^{2}=q_{1} q_{1}^{*}+q_{2} q_{2}^{*}
$$

is an invariant under an $S U(2)$ transformation. In Euclidean space:

$$
q_{Z}=q_{Y}=q_{Z}=1
$$

and

$$
q_{1} q_{1}^{*}+q_{2} q_{2}^{*}=3,
$$


a result which is approached asymptotically in the weak-field limit of general relativity [1-5].

Under a $S U(2)$ transformation:

$$
\left(\begin{array}{l}
q_{1}^{\prime} \\
q_{2}^{\prime}
\end{array}\right)=\left(\begin{array}{ll}
a & 0 \\
0 & a^{*}
\end{array}\right)\left(\begin{array}{l}
q_{1} \\
q_{2}
\end{array}\right),
$$

and this is a transformation of the metric spinor in the weak field limit. From Eqs. (39) and (42) it can be seen that the metric threevector $\mathbf{q}$ can always be represented as the metric two-spinor $q$ in threedimensional Euclidean space. Therefore, the corresponding metric four-vector $[1,4]$

$$
q^{\mu}=\left(q^{0}, \mathbf{q}\right)
$$

can always be represented as the metric three-spinor

$$
q^{\mu}=\left(q^{0}, q\right)
$$

in four-dimensional Euclidean spacetime, in which is defined the invariant

$$
\begin{aligned}
q^{\mu} q_{\mu} & =q^{02}-q_{X}^{2}-q_{Y}^{2}-q_{Z}^{2} \\
& =q^{02}-q_{1} q_{1}^{*}-q_{2} q_{2}^{*} .
\end{aligned}
$$

The Evans wave equation (4) in component form is

$$
\begin{aligned}
& (\square+k T) q^{0}=0, \\
& (\square+k T) \mathbf{q}=\mathbf{0},
\end{aligned}
$$

and thus there exists the equation

$$
(\square+k T)\left(\begin{array}{l}
q_{1} \\
q_{2}
\end{array}\right)=0,
$$

which the Evans equation for the two-spinor $q$.

In Euclidean spacetime, the metric three-vector components are defined as $[2,6]$

$$
q_{X}=\left|\frac{\partial \mathbf{R}}{\partial X}\right|, \quad q_{Y}=\left|\frac{\partial \mathbf{R}}{\partial Y}\right|, \quad q_{Z}=\left|\frac{\partial \mathbf{R}}{\partial Z}\right| .
$$

Consider now a region of non-Euclidean three-dimensional space such that each point is specified by three numbers $\left(u_{1}, u_{2}, u_{3}\right)$, the curvilinear 
coordinates [2]. The transformation equations between Cartesian and curvilinear coordinates are

$$
\begin{aligned}
& X=X\left(u_{1}, u_{2}, u_{3}\right), u_{1}=u_{1}(X, Y, Z) \\
& Y=Y\left(u_{1}, u_{2}, u_{3}\right), u_{2}=u_{2}(X, Y, Z), \\
& Z=Z\left(u_{1}, u_{2}, u_{3}\right), u_{3}=u_{3}(X, Y, Z),
\end{aligned}
$$

where the functions are single-valued and continuously differentiable. There is therefore a one-to-one correspondence between $(X, Y, Z)$ and $\left(u_{1}, u_{2}, u_{3}\right)$. The position vector in curvilinear coordinates in $\mathbf{R}\left(u_{1}, u_{2}, u_{3}\right)$. Define the arc length as the modulus of the infinitesimal displacement vector:

$$
d s=|d \mathbf{R}|=\left|\frac{\partial \mathbf{R}}{\partial u_{1}} d u_{1}+\frac{\partial \mathbf{R}}{\partial u_{2}} d u_{2}+\frac{\partial \mathbf{R}}{\partial u_{3}} d u_{3}\right|,
$$

where $\partial \mathbf{R} / \partial u_{i}$ is the metric coefficient whose modulus is the scale factor

$$
h_{i}=\left|\frac{\partial \mathbf{R}}{\partial u_{i}}\right| .
$$

The unit vectors of the curvilinear coordinate system may now be defined as

$$
\mathbf{e}_{(i)}=\frac{1}{h_{i}} \frac{\partial \mathbf{R}}{\partial u_{i}}, \quad i=1,2,3
$$

and we can write

$$
d \mathbf{R}=h_{1} d u_{1} \mathbf{e}_{(1)}+h_{2} d u_{2} \mathbf{e}_{(2)}+h_{3} d u_{3} \mathbf{e}_{(3)} .
$$

The unit vectors $\mathbf{e}_{(i)}$ are unit tangent vectors to the curve $u_{i}$ at point $P$, i.e., the three unit vectors of the curvilinear coordinate system are unit tangent vectors to the coordinate curves, are mutually orthogonal, and cyclically symmetric with $O(3)$ symmetry:

$$
\begin{gathered}
\mathbf{e}_{(1)} \cdot \mathbf{e}_{(2)}=0, \quad \mathbf{e}_{(1)} \cdot \mathbf{e}_{(3)}=0, \quad \mathbf{e}_{(2)} \cdot \mathbf{e}_{(3)}=0, \\
\mathbf{e}_{(1)} \times \mathbf{e}_{(2)}=\mathbf{e}_{(3)}, \quad \mathbf{e}_{(2)} \times \mathbf{e}_{(3)}=\mathbf{e}_{(1)}, \quad \mathbf{e}_{(3)} \times \mathbf{e}_{(1)}=\mathbf{e}_{(2)} .
\end{gathered}
$$

In the Euclidean limit the unit vectors $\mathbf{e}_{(i)}$ become the Cartesian unit vectors and the scale factors $h_{i}$ become the metric coefficients $q_{i}$. Therefore we arrive at the definition of the general three-vector field $\mathbf{F}$ in non-Euclidean three-space [2]:

$$
\mathbf{F}=F_{(1)} \mathbf{e}_{(1)}+F_{(2)} \mathbf{e}_{(2)}+F_{(3)} \mathbf{e}_{(3)}
$$


This definition is sketched below:

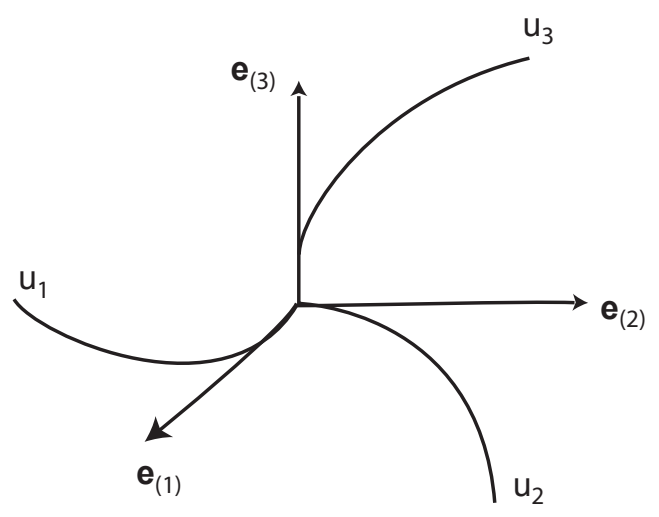

The metric three-vector in non-Euclidean three-space is therefore

$$
\mathbf{q}=q_{(1)} \mathbf{e}_{(1)}+q_{(2)} \mathbf{e}_{(2)}+q_{(3)} \mathbf{e}_{(3)},
$$

and similarly the metric four-vector in non-Euclidean four-dimensional spacetime is

$$
q^{\mu}=\left(q^{0}, \mathbf{q}\right) .
$$

The metric spinor in non-Euclidean three-space can always be defined through the relation

$$
q^{2}=q_{(1)}^{2}+q_{(2)}^{2}+q_{(3)}^{2}=q_{1} q_{1}^{*}+q_{2} q_{2}^{*},
$$

which is Eq. (39) in non-Euclidean three-space. We denote this metric spinor by

$$
q=\left(\begin{array}{l}
q_{1} \\
q_{1}
\end{array}\right)
$$

and it obeys the Evans equation

$$
(\square+k T) q=0
$$

in non-Euclidean three-space. Similarly the metric three spinor in fourdimensional non-Euclidean space is $\left(q^{0}, \mathbf{q}\right)$.

Define the parity operation in Euclidean three-space by

$$
\hat{P}(\mathbf{R})=-\mathbf{R}=-X \mathbf{i}-Y \mathbf{j}-Z \mathbf{k},
$$

i.e., the parity operation reverses the signs of $\mathbf{i}, \mathbf{j}, \mathbf{k}$, and therefore reverses the signs of the position vector $\mathbf{R}$ and the metric three-vector q:

$$
\hat{P}\left(\mathbf{q}_{X}\right)=-\left|\frac{\partial \mathbf{R}}{\partial X}\right| \mathbf{i}=-\mathbf{q}_{X}, \quad \text { etc. }
$$


Therefore, under parity,

$$
\hat{P}\left(q^{(R)}\right)=q^{(L)}
$$

Similarly, in non-Euclidean three-space, parity is defined as reversing the signs of the unit vectors $\mathbf{e}_{(1)}, \mathbf{e}_{(2)}, \mathbf{e}_{(3)}$ reversing the signs of $\mathbf{R}$ and $\mathbf{q}$ in non-Euclidean three-space, in which the parity operation produces, again:

$$
\hat{P}\left(q^{(R)}\right)=q^{(L)} .
$$

Therefore Eq. (63) is extended under parity to an equation in the fourspinor:

$$
(\square+k T) \psi=0,
$$

and this is the Evans equations for the four-spinor in non-Euclidean spacetime.

\section{DERIVATION OF THE DIRAC EQUATION OF SPECIAL RELATIVITY}

In the weak-field limit, Eq. (68) asymptotically approaches $[1,4]$

$$
k T \rightarrow \frac{k m}{V_{0}}=\frac{m^{2} c^{2}}{\hbar^{2}}, \quad\left(\square+\frac{m^{2} c^{2}}{\hbar^{2}}\right) \psi=0,
$$

and the Dirac equation of special relativity is deduced. In Eq. (69), $m$ is the rest mass of a particle and $V_{0}$ its rest volume. Equation (69) generalizes the Planck/de Broglie postulate to any particle (not only the photon), and also implies that the photon has a rest mass and rest volume. The rest frequency is defined by

$$
E_{0}=\hbar \omega_{0}=m c^{2}, \quad \omega_{0}=8 \pi c \ell^{2} / V,
$$

where

$$
\ell=\sqrt{G \hbar / c^{2}}
$$

is the Planck length. The product of the rest mass $m$ and the rest volume $V_{0}$ is a universal constant

$$
m V_{0}=\hbar^{2} k / c^{2}
$$

so every particle occupies a rest volume in nature, and there can be no point particles. This deduction is consistent with the fact that there are no singularities in nature, and no singularities in general relativity, a classical field theory. The Dirac Eq. (69) is well known to be an equation of relativistic quantum mechanics, which we have 
therefore deduced from classical general relativity using geometry. No probabilistic assumptions have been made in this derivation. However, it is well known [5] that the Dirac equation, unlike the Klein-Gordon equation, is consistent with the probabilistic interpretation of the wave function proposed by Bohr, Born, Heisenberg, and the Copenhagen School in natural philosophy. Neither the probabilistic interpretation of the wave function, nor the positivist speculations [6] of this School are required to deduce the Dirac equation from the novel Evans equation of classical general relativity. We see in Eq. (70) that the Evans equation also produces the wave particle dualism of de Broglie from geometry. Having deduced the Dirac equation, it is a straightforward to deduce from the Dirac equation the Schrödinger equation in the former's nonrelativistic limit. Having deduced the Schrödinger equation it then becomes possible in turn to deduce Newtonian dynamics in the classical limit of the Schrödinger equation $[5,7]$.

Therefore all the equations of linear quantum and classical dynamics can be deduced from non-linear Evans equation in well defined limits $[1,4]$. Noether's theorem can also be deduced $[1,3,4]$ from the non-Euclidean geometry used to construct the Evans equation, and therefore conservation laws of which the archetypical example is Newton's third law, can be deduced from the metric compatibility Eq. (1) and the Evans wave Eq. (4).

Another fundamental concept introduced by the Evans equation is that of rest curvature, which is the minimum curvature associated with any particle, such as the electron. The rest curvature is the inverse of the Compton wavelength squared:

$$
\left|R_{0}\right|=1 / \lambda^{2}=m^{2} c^{2} / \hbar^{2} .
$$

The rest curvature is therefore related to the rest energy in special relativity by

$$
E_{0}=m c^{2}=\hbar c \sqrt{\left|R_{0}\right|} .
$$

More generally, the quantum of energy for any particle (not only the photon) is given by

$$
E=\hbar c \sqrt{\left|R_{0}\right|}
$$

where

$$
|R|=\partial_{\mu} \Gamma_{\nu}^{\mu \nu}-\Gamma_{\nu}^{\mu \nu} \Gamma_{\mu \nu}^{\nu}
$$

in general relativity $[1,4,8]$. Here $\Gamma_{\mu \nu}^{\nu}$ is the Christoffel symbol. Therefore we arrive at the novel and fundamental law for all particles in general relativity there exists the relation

$$
E=\hbar \omega=\hbar c \sqrt{|R|},
$$

and this is a generalization of both the Planck-Law and the de Broglie wave-particle dualism to all particles in general relativity.

This new law is another consequence of the Evans wave Eq. (4), and is therefore a consequence of the geometrical constraint (1). 


\section{THE DIRAC MATRICES AS EIGENFUNCTIONS}

Dirac constructed his equation in about 1927 because the Schrödinger equation did not satisfy the requirements of relativity [7], in which the space and time coordinates must be developed as parts of a single spacetime. In relativity the relativistic momentum $\gamma m v$ and the energy are related by the Einstein equation

$$
E^{2}-p^{2} c^{2}=m^{2} c^{4}
$$

and this equation reduces to the Newtonian kinetic energy

$$
E=p^{2} / 2 m,
$$

when $p$ is small compared with $m c$ [7]. The operator replacement $[1,4,5]$

$$
p^{\mu}=i \hbar \partial^{\mu}, \quad p^{\mu}=(E / c, \mathbf{p}), \quad \partial^{\mu}=\left(\frac{1}{c} \frac{\partial}{\partial t},-\nabla\right)
$$

in Eq. (78) produces the Klein-Gordon equation

$$
\left(\square+m^{2} c^{2} / \hbar^{2}\right) q^{\mu}=0,
$$

to which the Evans Eq. (4) reduces in the limit (69). The KleinGordon equation produces a fundamental and well-known paradox $[5,7]$ if its eigenfunction is assumed to have a probabilistic ontology. The probability density from the Klein-Gordon equation can be negative (and therefore unphysical), whereas the probability density of the Schrödinger equation is always positive definite. Dirac therefore constructed an equation of the form

$$
\frac{\partial \psi}{\partial t}=\alpha_{X} \frac{\partial \psi}{\partial X}+\alpha_{Y} \frac{\partial \psi}{\partial Y}+\alpha_{Z} \frac{\partial \psi}{\partial Z}+\beta m c^{2}
$$

where $\alpha_{X}, \alpha_{Y}, \alpha_{Z}, \beta$ are constants [7]. The equation leads to a positive definite probability density. The four components of the Dirac equation must also be four Klein-Gordon equations, and the constants $\alpha_{X}, \alpha_{Y}, \alpha_{Z}, \beta$ must be $4 \times 4$ matrices, known as the Dirac matrices $\gamma^{\mu}$. The solutions of the Dirac equation separate at low kinetic energies into two doubly degenerate sets, one set is associated with positive kinetic and the other with negative kinetic energy, giving rise to anti-particles and the Dirac sea $[5,7]$. The degeneracy is removed by a magnetic field, giving rise from half integral spin to fermion spin resonance.

In order to derive the original form of the Dirac equation from the equivalent form (69) (the Klein-Gordon form of the Dirac equation), the d'Alembertian is expressed in terms of the anti-commutator of Dirac matrices [5]:

$$
=\frac{1}{2}\left\{\gamma^{\mu}, \gamma^{\nu}\right\} \partial_{\mu} \partial_{\nu}
$$


This equation implies

$$
\left\{\gamma^{\mu}, \gamma^{\nu}\right\}=2 q^{\mu \nu}
$$

i.e., in Euclidean spacetime the symmetric metric tensor $q^{\mu \nu}$ is half the commutator of the Dirac matrices. However we also know that the symmetric metric tensor is defined by [2]

$$
q^{\mu \nu}=\frac{1}{2}\left\{q^{\mu}, q^{\nu}\right\}
$$

and so

$$
\left\{\gamma^{\mu}, \gamma^{\nu}\right\}=\left\{q^{\mu}, q^{\nu}\right\}
$$

This equation shows that the Evans wave equation may be expressed as an eigenequation in the Dirac matrix generalized to non-Euclidean spacetime:

$$
(\square+k T) \gamma^{\mu}=0 .
$$

In the Euclidean limit

$$
\left(\square+m^{2} c^{2} / \hbar^{2}\right) \gamma^{\mu}=0 .
$$

In non-Euclidean spacetime, the elements of the $4 \times 4$ Dirac matrices are no longer unity but become space- and time-dependent quantities and the Dirac matrices in non-Euclidean spacetime are related to the tetrads [3] $q_{\mu}^{a}$. This result is consistent with the fact that the most general form of the Evans equation is an eigenequation in tetrads [1,4]:

$$
(\square+k T) q_{\mu}^{a}=0 .
$$

The Dirac matrix and spinor forms of the Dirac equation in general relativity are related by Eqs. (84) and (86).

The original form of the Dirac equation is obtained by substituting Eq. (83) into Eq. (69), which has been deduced in this paper from the Evans Eq. (4). This substitution produces:

$$
\left(\gamma^{\mu} \gamma^{\nu} \partial_{\mu} \partial_{\nu}+m^{2} c^{2} / \hbar^{2}\right) \psi=0
$$

Equation (90) may be obtained from the Dirac equation in its original form

$$
\left(i \gamma^{\mu} \partial_{\mu}-m c / \hbar\right) \psi=0 .
$$

The proof of this result is as follows:

Using the operator equivalence (80), Eq. (91) becomes

$$
\left(\gamma^{\mu} p_{\mu}-m c / \hbar\right) \psi=0 .
$$

Application of the operator $i \gamma^{\mu} \partial_{\mu}$ to Eq. (91) produces

$$
\left(-\gamma^{\mu} \partial_{\mu} \gamma^{\nu} \partial_{\nu}-i\left(\gamma^{\mu} \partial_{\mu}\right)(m c / \hbar)\right) \psi=0 .
$$


However, we know that

$$
i \gamma^{\mu} \partial_{\mu} \psi=(m c / \hbar) \psi
$$

and

$$
\partial_{\mu}[(m c / \hbar) \psi]=(m c / \hbar) \partial_{\mu} \psi,
$$

and so we obtain Eq. (90) from Eq. (91).

Equation (92), the original form of the Dirac equation, may now be expressed in the form

$$
\begin{aligned}
& (E+c \boldsymbol{\sigma} \cdot \mathbf{p}) q^{(L)}(\mathbf{p})=m c^{2} q^{(R)}(\mathbf{p}), \\
& (E-c \boldsymbol{\sigma} \cdot \mathbf{p}) q^{(R)}(\mathbf{p})=m c^{2} q^{(L)}(\mathbf{p}),
\end{aligned}
$$

which consists of two simultaneous equations [5] with

$$
q^{(L)}(\mathbf{0})=q^{(R)}(\mathbf{0})
$$

in the space and time dependent metric two-spinors. In the discussion to this paper it is shown that these spinors are obtained from the rest frame spinors by a Lorentz boost in special relativity.

Therefore all the major properties of the Dirac equation have been obtained from the Evans Eq. (4), which is therefore a generalized and nonlinear Dirac equation. The Evans equation also contains physics not present in the Dirac equation and therefore supplants the latter in natural philosophy.

\section{A FUNDAMENTAL PARADOX IN CLIFFORD ALGEBRA}

This paradox of Clifford algebra occurs in the standard method [5] of identifying the outer product of two Pauli spinors with the position vector $\mathbf{R}$ in the Pauli basis:

$$
H=\boldsymbol{\sigma} \cdot \mathbf{r}=\xi \xi^{+},
$$

where

$$
\boldsymbol{\sigma} \cdot \mathbf{r}=\left(\begin{array}{cc}
Z & X-i Y \\
X+i Y & -Z
\end{array}\right)
$$

and

$$
\xi \xi^{+}=\left(\begin{array}{ll}
\xi_{1} \xi_{1}^{*} & \xi_{1} \xi_{2}^{*} \\
\xi_{2} \xi_{1}^{*} & \xi_{2} \xi_{2}^{*}
\end{array}\right) .
$$


The standard method in Clifford algebra [5] expresses the outer product $\xi \xi^{+}$as a traceless unitary matrix by noting that Eqs. (23) to (26) can be written as

$$
\begin{aligned}
\xi_{1}^{\prime} & =a \xi_{1}+b \xi_{2}, \\
\xi_{2}^{\prime} & =-b^{*} \xi_{1}+a^{*} \xi_{2}, \\
-\xi_{2}^{* \prime} & =a\left(-\xi_{2}^{*}\right)+b \xi_{1}^{*}, \\
\xi_{1}^{* \prime} & =-b^{*}\left(-\xi_{2}^{*}\right)+a^{*} \xi_{1}^{*} .
\end{aligned}
$$

Therefore the Pauli spinor $\left(\begin{array}{l}\xi_{1} \\ \xi_{2}\end{array}\right)$ transform in the same way as $\left(\begin{array}{c}-\xi_{2}^{*} \\ \xi_{1}^{*}\end{array}\right)$. In shorthand notation,

$$
\xi \sim \zeta \xi^{*}, \quad \zeta=\left(\begin{array}{cc}
0 & -1 \\
1 & 0
\end{array}\right) .
$$

In other words, we can think of

$$
\xi_{1} \rightarrow-\xi_{2}^{*}, \quad \xi_{1}^{\prime} \rightarrow-\xi_{2}^{* \prime}, \quad \xi_{2} \rightarrow \xi_{1}^{*}, \quad \xi_{2}^{\prime} \rightarrow \xi_{1}^{* \prime} .
$$

Similarly, we can write Eqs. (9) to (12) as

$$
\begin{aligned}
\xi_{1}^{* \prime} & =\xi_{1}^{*} a^{*}+\xi_{2}^{*} b^{*}, \\
\xi_{2}^{* \prime} & =-\xi_{1}^{*} b+\xi_{2}^{*} a, \\
\xi_{2}^{\prime} & =\xi_{2} a^{*}-\xi_{1} b^{*}, \\
-\xi_{1}^{\prime} & =-\xi_{2} b-\xi_{1} a,
\end{aligned}
$$

so we can think of

$$
\xi_{1}^{*} \rightarrow \xi_{2}, \quad \xi_{1}^{* \prime} \rightarrow \xi_{2}^{\prime}, \quad \xi_{2}^{*} \rightarrow-\xi_{1}, \quad \xi_{2}^{* \prime} \rightarrow-\xi_{1}^{\prime} .
$$

From Eqs. (107) and (112),

$$
\xi \xi^{+} \sim\left(\begin{array}{c}
-\xi_{2}^{*} \\
\xi_{1}^{*}
\end{array}\right)\left(-\xi_{1}, \xi_{2}\right)=\left(\begin{array}{cc}
\xi_{2}^{*} \xi_{1} & -\xi_{2}^{*} \xi_{2} \\
-\xi_{1} \xi_{1}^{*} & \xi_{1}^{*} \xi_{2}
\end{array}\right) .
$$

The standard method [5], however, asserts that

$$
\xi^{+} \sim(\zeta \xi)^{T}=\left(-\xi_{2}, \xi_{1}\right) .
$$


This method does not agree with the fact that $\xi^{+}$transforms as $\left(-\xi_{1}, \xi_{2}\right)$, from Eqs. (112) and (107). However, if, for the sake of continuity, one accepts the incorrect Eq. (114), one obtains

$$
H=h=\xi \xi^{+}=\left(\begin{array}{cc}
-\xi_{1} \xi_{2} & \xi_{1}^{2} \\
-\xi_{2}^{2} & \xi_{1} \xi_{2}
\end{array}\right)=\left(\begin{array}{cc}
Z & X-i Y \\
X+i Y & -Z
\end{array}\right)
$$

that is,

$$
\begin{aligned}
X-i Y & =-\xi_{1}^{2}, \\
X+i Y & =\xi_{2}^{2}, \\
Z & =\xi_{1} \xi_{2} .
\end{aligned}
$$

Multiplication of Eqs. (116) and (117) produces

$$
X^{2}+Y^{2}=(X-i Y)(X+i Y)=-\xi_{1}^{2} \xi_{2}^{2} ;
$$

and, adding Eqs. (118) and (119),

$$
X^{2}+Y^{2}+Z^{2}=0
$$

an equation that produces the paradox

$$
X=Y=Z \stackrel{?}{=} 0
$$

because $X, Y$ and $Z$ are all real-valued.

This fundamental paradox is also present for any type of outer product of Pauli spinors. For example, if we attempt to identify

$$
\left(\begin{array}{cc}
Z & X-i Y \\
X+i Y & -Z
\end{array}\right)=\left(\begin{array}{cc}
\xi_{1} \xi_{2}^{*} & -\xi_{2} \xi_{2}^{*} \\
-\xi_{1} \xi_{1}^{*} & \xi_{2} \xi_{1}^{*}
\end{array}\right)
$$

we find

$$
\begin{aligned}
X-i Y & =-\xi_{2} \xi_{2}^{*}, \\
X+i Y & =-\xi_{1} \xi_{1}^{*}, \\
Z & =\xi_{1} \xi_{2}^{*}=-\xi_{2} \xi_{1}^{*} .
\end{aligned}
$$

Multiplication of Eqs. (123) and (124) gives

$$
X^{2}+Y^{2}=\xi_{1} \xi_{2}^{*} \xi_{2} \xi_{1}^{*}
$$

and, adding Eqs. (125) and (126), we obtain Eqs. (120) and (121) again.

Therefore this paradox is a basic paradox of Clifford algebra because it occurs in the basic relation between three-vector elements and two-spinor elements. We avoid using the paradox in this paper by devising Eq. (28) by identifying the inner product of Pauli spinors with the inner product of three vectors. 


\section{DISCUSSION}

In this paper we have derived the fundamental properties of the Dirac equation from the metric compatibility condition (1) of non-Euclidean geometry. There remains only the problem of relating the Dirac equation to the Lorentz boost of special relativity [5]. The original method, due to Ryder [5], relies solely on the Lorentz boosts:

$$
\begin{aligned}
& q^{(R)}(\mathbf{p})=\exp \left(-i \frac{\boldsymbol{\sigma}}{2} \cdot \boldsymbol{\phi}\right) q^{(R)}(\mathbf{0}) \\
& q^{(L)}(\mathbf{p})=\exp \left(i \frac{\boldsymbol{\sigma}}{2} \cdot \boldsymbol{\phi}\right) q^{(L)}(\mathbf{0})
\end{aligned}
$$

These are generated from each other by the parity operator. Solving Eqs. (127) and (128) simultaneously leads to the result

$$
\begin{aligned}
& (E-c \boldsymbol{\sigma} \cdot \mathbf{p}) q^{(R)}(\mathbf{p})-m c^{2} q^{(L)}(\mathbf{p}) \\
= & (E+c \boldsymbol{\sigma} \cdot \mathbf{p}) q^{(L)}(\mathbf{p})-m c^{2} q^{(R)}(\mathbf{p}) .
\end{aligned}
$$

Comparison with Eqs. (96) and (97) shows that the Dirac equation is a special case of Eq. (129). The Ryder method does not, however, unequivocally obtain the Dirac equation from Eq. (129). In order to try to achieve this result we have to consider the extra condition

$$
\begin{aligned}
q^{(R)}(\mathbf{p}) q^{(L) T}(\mathbf{p}) & =q^{(R)}(0) q^{(L) T}(0)=q^{(R)}(0) q^{(R) T}(0) \\
& =q^{(L)}(0) q^{(R) T}(0)=q^{(L)}(0) q^{(L) T}(0)
\end{aligned}
$$

which is obtained from Eq. (98). Equation (130) implies

$$
\begin{aligned}
& q^{(R)}(\mathbf{p}) q^{(L) T}(\mathbf{p})=q^{(R)}(\mathbf{p}) q^{(R) T}(\mathbf{p}) \\
= & q^{(L)}(\mathbf{p}) q^{(R) T}(\mathbf{p})=q^{(L)}(\mathbf{p}) q^{(L) T}(\mathbf{p}) .
\end{aligned}
$$

Multiplying Eq. (129) on both sides by $q^{(L) T}$, we obtain

$$
\begin{aligned}
& (E-c \boldsymbol{\sigma} \cdot \mathbf{p}) q^{(R)} q^{(L) T}-m c^{2} q^{(L)} q^{(L) T} \\
= & (E+c \boldsymbol{\sigma} \cdot \mathbf{p}) q^{(L)} q^{(L) T}-m c^{2} q^{(R)} q^{(L) T}
\end{aligned}
$$

and, using Eq. (131),

$$
E-c \boldsymbol{\sigma} \cdot \mathbf{p}-m c^{2}=E+c \boldsymbol{\sigma} \cdot \mathbf{p}-m c^{2},
$$


which implies

$$
\begin{aligned}
\mathbf{p} & =\mathbf{0}, \\
E-m c^{2} & =E-m c^{2} .
\end{aligned}
$$

This is the maximum amount of information obtainable from the Lorentz boost, so the Dirac Eq. (92) cannot be obtained unequivocally from a Lorentz boost. The correct way of deducing the Dirac equation unequivocally is from general relativity, as in this paper.

Acknowledgments. The Ted Annis Foundation and Craddock Inc. are acknowledged for funding and the Fellows and Emeriti of AIAS for many interesting discussions.

\section{REFERENCES}

1. M. W. Evans, Found Phys. Lett. 16, 367 (2003); in press (December 2003); www.aias.us.

2. E. G. Milewski, ed., Vector Analysis Problem Solver (Research and Education Association, New York, 1987).

3. S. M. Carroll, Lecture Notes in General Relativity (University of California, Santa Barbara, graduate course on arXiv:grqe/9712019vl, 3 December 1997).

4. M. W. Evans, Found Phys. Lett., in press (2004).

5. L. H. Ryder, Quantum Field Theory (University Press, Cambridge, 1987, 1996).

6. M. Sachs and A. R. Roy, eds., Mach's Principle and the Origin of Inertia (Apeiron, Montreal, 2003).

7. P. W. Atkins, Molecular Quantum Mechanics (University Press, Oxford, 1983). 